\title{
LIQUID PHASE CONTAINED IN POROUS ROCK AS OBSERVED BY PROTON MAGNETIC RELAXATION
}

\author{
H. HARAŃCZYK ${ }^{a}$ AND A. WóJCIK ${ }^{b}$ \\ a Institute of Physics, Jagiellonian University, Reymonta 4, 30-059 Cracow, Poland \\ ${ }^{b}$ Faculty of Geology, Geophysics and Environmental Protection, Dept. of Geophysics \\ Academy of Mining and Metalurgy, Cracow, Poland
}

(Received February 14, 2000; revised version April 28, 2000)

\begin{abstract}
High power proton relaxometry was applied to investigate the liquid phase contained in porous rock. Proton free induction decays and spin-lattice relaxation times allowed us to investigate the pore distribution and the contribution of mobile and of immobilized liquid. The differences in pore distributions in oil-containing limestone and in Weglowicki sandstone were found. The fractal exponent for pore distribution in Węglowicki sandstone was fitted using both stretched exponential and modified stretched exponential models. The results of both approaches are compared.
\end{abstract}

PACS numbers: $81.05 . \mathrm{Rm}, 76.60 . \mathrm{s}$

\section{Introduction}

Recently proton magnetic relaxation is successfully applied to investigate liquid fraction contained in porous systems. It supplies information on water bound on the surface of controlled pore glass [1] or on water filling the whole pore volume [2-4]; as well as on the process of cement paste hydration [5]. In geophysics, proton magnetic relaxation allows one to evaluate the several petrophysical parameters as: porosity [6], permeability [6, 7], content of water and hydrocarbons $[8,9]$, differentiation of mobile or bound liquid phase $[10,11]$ in porous rock. However, till now the analysis of proton spin-lattice relaxation times distribution was emphasized. The analysis of free induction decays (FIDs) was treated with less attention [12].

The aim of our paper is to observe the liquid fraction present in oil-containing Węglowicki sandstone and in limestone using spin-grouping method [13] which supplies two-dimensional analysis of the proton spin-lattice relaxation function versus proton free induction decays. The samples obtained from the different depths of the same borehole as well as the samples from the different sites of the same core were measured. 


\section{Materials and methods}

Table I shows an absolute content of hydrocarbons in oil-containing porous limestone samples $\left(\mathrm{Ca}_{2}\right)$ from "Wysoka Kamienicka-22" borehole (depth $3055.5 \mathrm{~m}$ ). These numbers may vary within the core volume because of rock composition inhomogeneities.

\section{TABLE I}

The content of carbohydrates in oil-containing limestone.

\begin{tabular}{l|c|c}
\hline & sample '71' & sample '72' \\
\hline total content of HC [mg HC/rock] & 7.8 & 10 \\
mobile HC [mg HC/rock] & 5.3 & 2.5 \\
mobile HC [\%] & 68.0 & 66.2 \\
resident HC [mg HC/rock] & 6.7 & 3.4 \\
resident HC [\%] & 32.0 & 33.8
\end{tabular}

The samples of oil-containing Weglowicki sandstone were obtained from the "Węglówka" borehole (depth 551-555 m, sample "we1" from the height of 70-80 cm as measured from the bottom of the core and the sample "we3" from 120-130 cm of the core; and from the depth of 547-551 m, 40-50 cm from the bottom of the core-sample "we2").

Proton spin-lattice relaxation times were measured on a fully computer controlled spectrometer WNS-65 (Waterloo NMR Spectrometers, St. Agatha, Ontario, Canada) working at the resonance frequency of $30 \mathrm{MHz}$. Transmitter power was $400 \mathrm{~W}$; pulse lengths $\pi / 2=1.2 \mu$ s for sandstone and $\pi / 2=3.4 \mu \mathrm{s}$ for limestone samples. The spectrometer was controlled by an IBM 80486 clone, the data were acquired using a Compuscope 2000 card. Free induction decays were averaged over 2000 accumulations. Proton spin-lattice relaxation times were measured using the saturation method $(\pi / 2-\tau-\pi / 2)$ for limestone and the inversion recovery method $(\pi-\tau-\pi / 2)$, sampling the FID for different times $t$ after $\pi / 2$ pulse. The data were averaged from 400 accumulations. The repetition time was $5 \mathrm{~s}$. The temperature $t=25^{\circ} \mathrm{C}$ was stabilized in a gaseous nitrogen stream with the accuracy of $0.1^{\circ} \mathrm{C}$.

The obtained data were analyzed using the program CracSpin (Jagiellonian University, Cracow, Poland) which performs the two-dimensional data decomposition in time domain [13].

\section{Results and discussion}

For three samples of Węglowicki sandstone proton free induction decays were recorded (FID for the sample "we2" is shown in Fig. 1). In all the samples FID is well described by a superposition: Gaussian function coming from the solid proton fraction (component "1") and exponentially decaying signal coming from the mobile liquid proton fraction (component "2"). The fitted values of $T_{2}^{*}$ are shown in Table IIa. The $T_{2}^{*}$ value defined for the Gaussian component as a time 

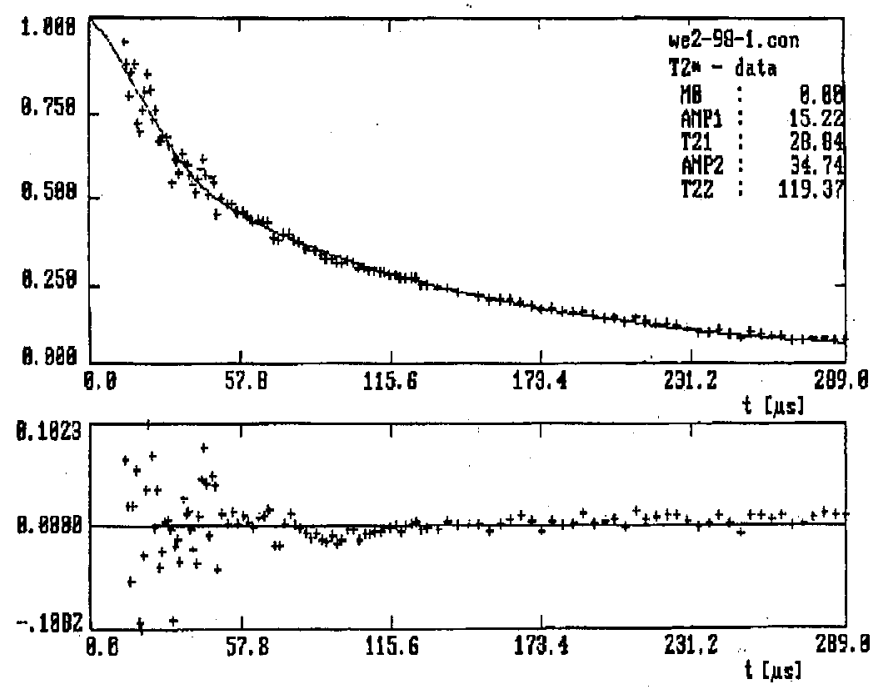

Fig. 1. Proton free induction decay for Węglowicki sandstone, sample "we2" (+). The superposition of Gaussian and single exponential line is fitted (solid line). The lower plot shows the residual function for this fit.

TABLE II

Proton free induction decays for Węglowicki sandstone (a) and for limestone (b) recorded at room temperature. For the Gaussian component the $T_{2}^{*}$ value was taken as the time at which the signal relaxes to $1 / e$ of its initial magnitude. For the limestone samples the intermediate component ("2") was fitted as Gaussian, however, the exponential fitting did not differ very much in amplitude and the time constant. The amplitudes $A_{i}$ are normalized to the total amplitude of the signal.

\begin{tabular}{|c|c|c|c|c|c|c|}
\hline \multirow[t]{2}{*}{ (a) } & \multicolumn{2}{|c|}{ component "1" } & \multicolumn{2}{|c|}{ component " 2 " } & & \\
\hline & $A_{1}$ & $\mathcal{T}_{2}^{*}[\mu \mathrm{s}]$ & $A_{2}$ & $T_{2}^{*}[\mu \mathrm{s}]$ & & \\
\hline sample "we1" & $31.7 \%$ & 24.26 & $69.3 \%$ & 100.0 & & \\
\hline sample "we2" & $30.5 \%$ & 28.84 & $69.5 \%$ & 119.4 & & \\
\hline sample "we3" & $60.2 \%$ & 24.02 & $39.8 \%$ & 88.6 & & \\
\hline \multirow[t]{2}{*}{ (b) } & \multicolumn{2}{|c|}{ component "1" } & \multicolumn{2}{|c|}{ component " 2 " } & \multicolumn{2}{|c|}{ component " 3 " } \\
\hline & $A_{1}$ & $T_{2}^{*}[\mu \mathrm{s}]$ & $A_{2}$ & $T_{2}^{*}[\mu \mathrm{s}]$ & $A_{3}$ & $T_{2}^{*}[\mu \mathrm{s}]$ \\
\hline sample ' 71 ' & $55.0 \%$ & 15.3 & $29.4 \%$ & 55.6 & $15.5 \%$ & 297 \\
\hline sample ' 72 ' & $60.4 \%$ & 14.3 & $28.9 \%$ & 30.22 & $16.6 \%$ & 163 \\
\hline
\end{tabular}

needed for the signal to relax to the $1 / e$ of its initial magnitude allows the direct comparison between the time constants calculated for liquid (exponential) and solid (Gaussian) components independently of the fitted function used.

The meaningful contribution of the Gaussian component in Węglowicki sandstone is worth mentioning. It may come from the solid fraction of oil present in 
porous rock, i.e. mineral wax as well as from $\mathrm{OH}$ groups and crystalization water trapped in pores of mineral matrice.

Proton free induction decays measured for high power NMR pulses allows one to observe the total proton signal. However, because of $B_{0}$ field inhomogeneities (for water $T_{2}^{*} \cong 12 \mathrm{~ms}$ ), the precise fit of several liquid components [6] is not possible, as the measured $T_{2}^{*}$ is given by

$$
\frac{1}{T_{1}^{*}}=\frac{1}{T_{2}}+\frac{\gamma \Delta B_{0}}{2}
$$

where the contribution of $B_{0}$ inhomogeneities overwhelms the observed effect for the components which relax with long $T_{2}$. Thus, it is possible that in the case of limestone both immobilized and mobile liquid carbohydrates contribute to the averaged liquid proton subsystem.

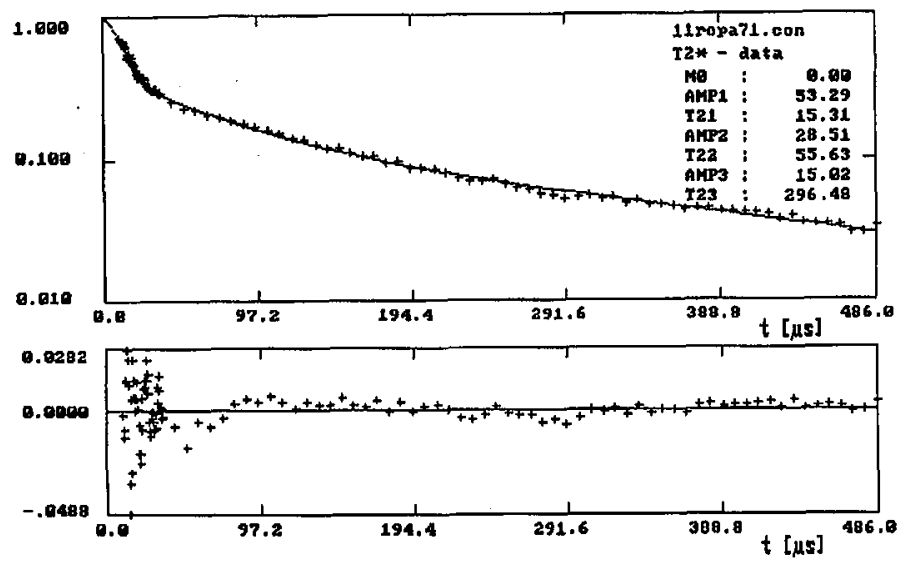

Fig. 2. Proton free induction decay for oil-containing limestone, sample ' 71 ' $(+)$. The superposition of Gaussian, "intermediate" component (Gaussian) and single exponent is fitted (solid line). The residual function for the so defined fit is presented on the lower plot.

The FID signal from oil-containing limestone (Fig. 2) differs from the FID signal recorded for Węglowicki sandstone. Beside the Gaussian component coming from the solid component of the proton signal and the exponentially decaying proton signal from the mobile liquid fraction, there is an intermediate signal, which may be fitted both by very broad Gaussian and an exponent. The obtained fits of $T_{2}^{*}$ and amplitude values are shown in Table IIb. As in sandstone, the solid signal may come from the organic solid fraction present in pores, i.e. mineral wax, or from $\mathrm{OH}$ groups and crystallization water trapped in pores of rock. One cannot exclude, however, that the observed solid signal comes from the protons bound inside rocky matrix. The "intermediate" signal fraction (characterized by $T_{2}^{*}$ of the order of $45 \mu \mathrm{s}$ - as averaged over both measured samples) most likely may be assigned to the solid proton fraction.

In contrast to NMR results, the contributions of mobile and resident proton fraction in the examined limestone samples (Table I) were similar. However, this 
can be caused by the inhomogeneities of the rock. Thus, the detailed analysis of direct correlations between gravimetric data and the magnitudes of the FID signal was not performed.

To get the information on the pore distribution, proton spin-lattice relaxation functions were recorded.

For both samples of limestone the spin-lattice relaxation function was fitted by a single exponential function with a sufficient quality (Fig. 3). The results of the fits are presented in Table IIIa. In $T_{1}$ measurements (using the program CracSpin performing the two-dimensional data analysis in time domain) for limestone the intermediate FID component was not recorded. This was caused by a lesser ( 400 per one FID only) number of accumulations and, thus, by a lower signal-to-noise ratio.

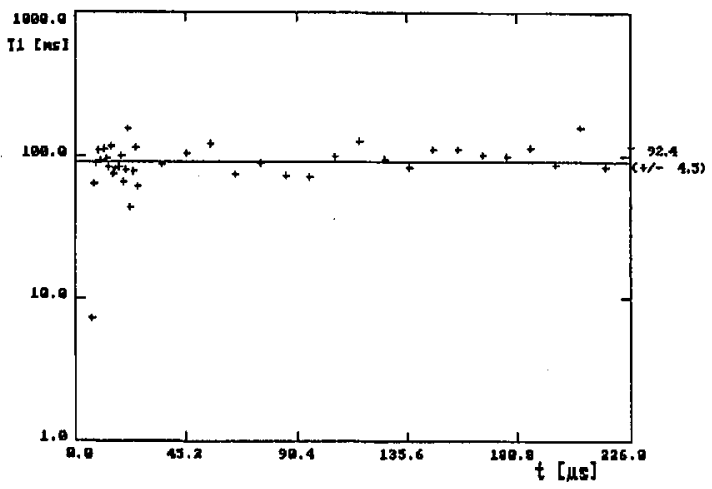

Fig. 3. Proton spin-lattice relaxation times for limestone sample ' 71 ' calculated as a function of time $t$ for the FID signal; the fits were obtained using the spin-grouping method.

\section{TABLE III}

Proton spin-lattice relaxation times measured for oil-containing limestone. The lesser number of accumulations for FIDs (400) excluded a very fine analysis of FID (see Table IIb); single-exponential fits (a); the fit of the stretched exponential function (b).

\begin{tabular}{c|c|c|c|c|c|c}
\hline \hline (a) & & $T_{1}[\mathrm{~ms}]$ & $A_{1}$ & $T_{2}^{*}[\mu \mathrm{s}]$ & $A_{2}$ & $T_{2}^{*}[\mu \mathrm{s}]$ \\
\hline sample '71' & & 92.4 & $70.9 \%$ & 12.9 & $29.1 \%$ & 92.4 \\
sample '72' & & 186.4 & $79.3 \%$ & 17.2 & $20.7 \%$ & 186.4 \\
\hline (b) & $\alpha$ & $T_{\mathrm{SE}}[\mathrm{ms}]$ & $A_{1}^{\mathrm{SE}}$ & $T_{2}^{*}[\mu \mathrm{s}]$ & $A_{2}^{\mathrm{SE}}$ & $T_{2}^{*}[\mu \mathrm{s}]$ \\
\hline sample '71' & 0.90 & 88.8 & $70.9 \%$ & 12.9 & $29.1 \%$ & 145 \\
sample '72' & 0.90 & 214 & $79.4 \%$ & 17.4 & $20.6 \%$ & 114
\end{tabular}

The single exponential form of proton spin-lattice relaxation function may suggest the presence of fast proton exchange between the pores of rock matrix. However, it may also reflect the narrow distribution of relaxation times for protons 
in isolated pores of a similar size. In the second case, the close values of relaxation times are not resolved by the fitting procedure. To test this hypothesis we fitted the stretched exponential (SE) function. Such function describes the fractal distribution of pores [14]

$$
F(\tau)=A^{\mathrm{SE}} \exp \left[-\left(\frac{\tau}{T_{\mathrm{SE}}}\right)^{\alpha}\right]
$$

The stretched exponential fits are shown in Table IIIb. Either the values of $T_{1}, T_{2}^{*}$ or the amplitude values are close to the ones fitted using the single exponential fits. The fractal exponent $\alpha$ is close to 1 . A similar value was observed for water in controlled pore glasses [4] which reflected the narrow distribution of pore sizes and the narrow range of self-similarity of the system. We suggest that it is the case for our oil-containing sandstone samples.

In contrast to limestone, in Węglowicki sandstone neither a single exponential function nor a double exponential function was fitted with a sufficient accuracy to proton spin-lattice relaxation. Two-dimensional spectroscopy in time domain supplies the information on a spin-lattice relaxation function as a function of time $t$ after $\pi / 2$ pulse [13]. The spin-lattice relaxation function for Węglowicki sandstone (sample "we2") measured at $t=49 \mu \mathrm{s}$ and fitted using the stretched exponential function is shown in Fig. 4.

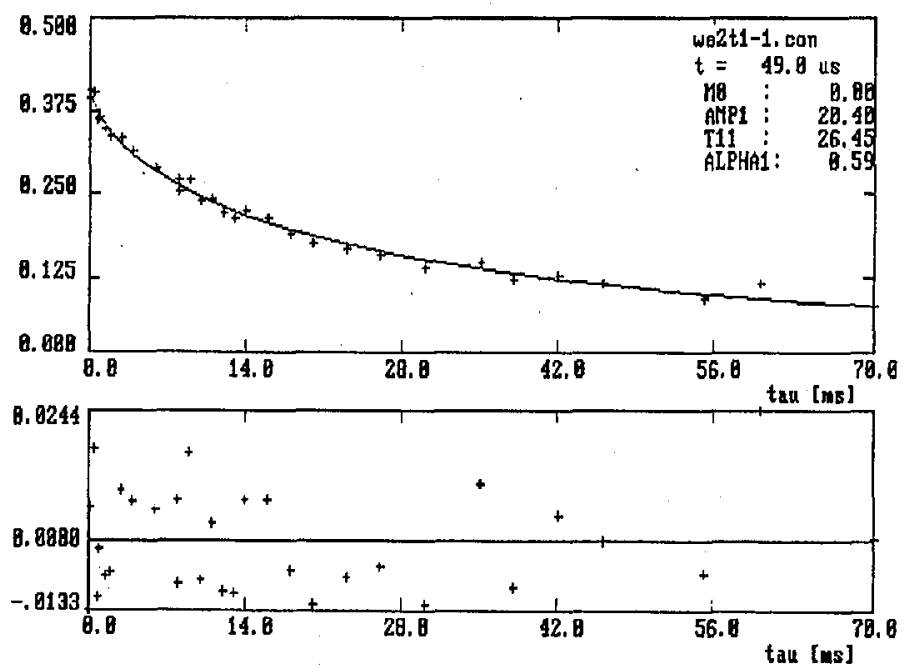

Fig. 4. The normalized proton spin-lattice relaxation for Weglowicki sandstone sample "we2" (+) recorded at $49 \mu$ s after $\pi / 2$ pulse and the stretched exponential function (solid line) fitted to the data. The lower plot shows the residual function.

For the stretched exponential fit the dependency of $T_{1}$ and the fractal exponents $\alpha$ versus time $t$ is shown in Fig. 5, whereas Table IV presents the obtained averaged values of $\alpha$ and $t$.

The disadvantage of the stretched exponential function is an artificial overestimation of the signal component populations as compared with the popula- 


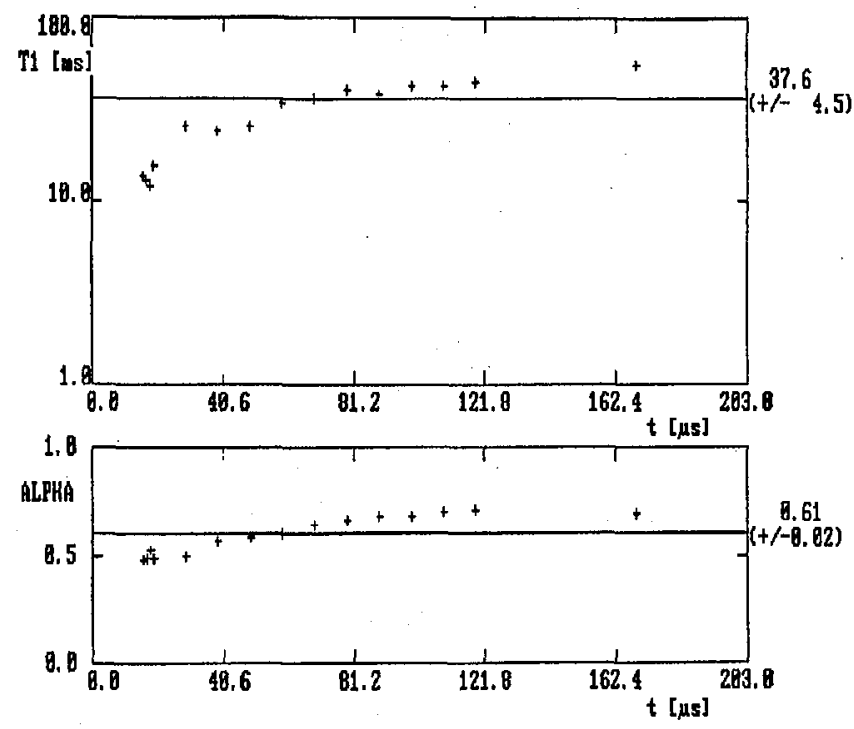

Fig. 5. The values of relaxation times $T_{1}$ and fractal exponent $\alpha$ of the stretched exponential fitted to the proton spin-lattice relaxation function for Węglowicki sandstone, sample "we2", fitted as a function of time $t$ after $\pi / 2$ pulse.

\section{TABLE IV}

Proton spin-lattice relaxation times $T_{1}$ measured for Węglowicki sandstone, sample "we2". In our system, the inversion recovery method requires that the circuit of shortening the spectrometer dead time is not used. Thus, the artificial broadening and the increase in magnitude of the fitted Gaussian function was observed.

\begin{tabular}{c|c|c|c|c|c}
\hline \hline$\alpha$ & $T_{1}[\mathrm{~ms}]$ & $A_{1}^{\mathrm{SE}}$ & $T_{2}^{*}[\mu \mathrm{s}]$ & $A_{2}^{\mathrm{SE}}$ & $T_{2}^{*}[\mu \mathrm{s}]$ \\
\hline $0.61 \pm 0.02$ & $37.6 \pm 4.5$ & $49.5 \%$ & 33.5 & $50.5 \%$ & 138.1
\end{tabular}

tions of components described by a single exponential function. This is implied by a non-physical, infinite value of the first derivative of the stretched exponential function for $\tau \rightarrow 0$

$$
\frac{\mathrm{d} A^{\mathrm{SE}}(\tau)}{\mathrm{d} \tau} \rightarrow-\frac{\alpha A_{0}^{\mathrm{SE}}}{T_{\mathrm{SE}}^{\alpha}}\left(\frac{1}{\tau}\right)^{1-\alpha} \rightarrow-\infty
$$

In contrary, in every physical system there is a minimal non-zero relaxation time, which cuts the relaxation time distribution and, thus, the value of relaxation function first derivative has a finite value. Peyron et al. [15] overcome this limitation proposing a fenomenological formula for a modified stretched exponential (MSE)

$$
A^{\mathrm{MSE}}(\tau)=A_{0}^{\mathrm{MSE}} \exp \left[-\frac{\tau}{T_{0}}\left(1+\frac{\tau}{T_{\mathrm{C}}}\right)^{\beta-1}\right]
$$

which exhibits a correct long-and short-time behaviour. 
In a short-time regime $\left(\tau \ll T_{\mathrm{C}}\right)$, MSE model has a desired single exponential behaviour

$$
A^{\mathrm{MSE}}(\tau) \rightarrow A_{0}^{\mathrm{MSE}} \exp \left(-\frac{\tau}{T_{0}}\right)
$$

whereas in a long-time approximation $\left(\tau \gg T_{\mathrm{C}}\right)$, it presents the limiting stretched exponential behaviour

$$
A^{\mathrm{MSE}}(\tau) \rightarrow A_{0}^{\mathrm{MSE}} \exp \left[-\left(\frac{\tau}{T_{\mathrm{MSE}}}\right)^{\beta}\right]
$$

where $T_{\mathrm{MSE}}=T_{0}^{1 / \beta} T_{\mathrm{C}}^{1-1 / \beta}$.

Both models used to fit the data for Węglowicki sandstone yielded very close values of parameters. Namely, the value of the time constant supplied by both models

$$
\chi=\frac{T_{\mathrm{MSE}}}{T_{\mathrm{SE}}} \quad \text { is } \quad \bar{\chi}=1.011 \text { and } \sigma_{\chi}=0.069[16] .
$$

The artificial magnification of the amplitude caused by the stretched exponential model

$$
\delta=\frac{A^{\mathrm{SE}}-A^{\mathrm{MSE}}}{A^{\mathrm{MSE}}}
$$

was equal to 0.035 with $\sigma_{\delta}=0.020$.

Additionally, in Węglowicki sandstone the effect of the artificial magnification of the amplitude was still small as the whole spin-lattice relaxation function was well described by one stretched exponential function and the proton spin subsystems were differentiated by the different components of free induction decay function.

The obtained value of fractal exponent significantly differs from 1 and is $\alpha=0.61 \pm 0.01$ for times $t>30 \mu \mathrm{s}$, whereas for shorter times $t$ it is equal to $0.51 \pm 0.01$.

This is the effect observed in the case of presence of broad fractal distribution of pores of oil-containing rock; the differentiation of mobile and immobilized liquid fraction should be understood not as a signal coming from two well-defined groups of pores but coming from different spin subsystems from every given pore.

Usually the stretched exponential fits are very effective, however, by their effectiveness they may mask the case where the relaxation function is a superposition of several exponentials. The CracSpin fitting program yields multiexponential fits which may be compared with the stretched exponential fits. This allows one, with a high dose of credibility, to discover the real form of relaxation function.

In the case of Weqglowicki sandstone, a high quality of fits clearly suggests that the proton spin-lattice relaxation function is in a stretched exponential form, whereas for oil-containing limestone it was well described by a single exponential function. This suggests that in the case of limestone samples, the close-to-uniform distribution of pore sizes occurs, whereas, in the case of Weglowicki sandstone, the pore distribution is well approximated by the self-scaling distribution [14].

The preliminary results presented here suggest that depending on the kind or origin of a porous rock sample the relaxation function may be described either 
by a stretched exponential or by a multiexponential function. The next step of our research will be the explanation of the solid component of the proton signal. If it comes from the protons bound in rocky matrix not from the protons of oil, it may be used as an independent standard to evaluate the content of oil in porous rock.

\section{References}

[1] H. Harańczyk, K.G. Soga, R.J. Rumm, M.M. Pintar, Magn. Reson. Imaging 9, 723 (1991).

[2] C. Choi, H. Harańczyk, K.G. Soga, R.J. Rumm, M.M. Pintar, J. Appl. Phys. 80, $5861(1996)$.

[3] F. D’Orazio, J.C. Tarczon, W.P. Halperin, K. Eguchi, T. Mizusaki, J. Appl. Phys. 65, $742(1989)$.

[4] K.G. Soga, H. Harańczyk, R.J. Rumm, M.M. Pintar, Magn. Reson. Imaging 9, 727 (1991).

[5] R. Rumm, H. Harańczyk, H. Peemoeller, M.M. Pintar, Cement \& Concrete Res. 21, 391 (1991).

[6] A. Timur, J. Petroleum Technol. 21, 775 (1969).

[7] J.D. Loren, J. Petroleum Technol. 24, 923 (1972).

[8] R.L. Kleinberg, W.E. Kenyon, P.P. Mitra, J. Magn. Reson. A 108, 206 (1994).

[9] J.D. Robinson, J.D. Loren, E.A. Vajnar, D.E. Hartman, J. Petroleum Technol. 26, 226 (1974).

[10] G. Gubelin, A. Boyd, J. Petroleum Technol. 49, 718 (1997).

[11] H.L. McKague, J.R. Hearst, R.L. Ward, N.R. Burkhard, Nucl. Geophys. 6, 359 (1992).

[12] W.E. Kenyon, Nucl. Geoph. 6, 153 (1992).

[13] W. Węglarz, H. Harańczyk, in: Mat. XXVI Ogólnopol. Sem. MRJ, Kraków 1993, Institute of Physics, Report No. 1658/PL, Kraków 1994, p. 137 (in Polish).

[14] C. DeDominicis, H. Orland, F. Lainee, J. Phys. Lett. 46, L-463 (1985).

[15] M. Peyron, G.K. Pierens, A.J. Lucas, L.D. Hall, R.C. Steward, J. Magn. Reson. A 118, 214 (1996).

[16] H. Harańczyk, A. Wójcik, Mol. Phys. Rep., in press. 